\title{
Awareness and perception of vitamin D and vitamin D fortified foods in the North East of England
}

Vitamin D plays an essential role in the maintenance of bone health and in the prevention and treatment of a larger array of diseases $^{(1)}$. Vitamin D deficiency has increased in recent years, with the UK, and particularly the North-East region, more at risk, largely due to their latitude ${ }^{(2)}$. Adequate dietary intakes are therefore essential in maintaining optimal vitamin D levels. Natural dietary sources of vitamin D are not consumed in sufficient quantities to sustain levels ${ }^{(1)}$, therefore fortified foods have a crucial role in maintaining vitamin D sufficiency ${ }^{(2)}$. As public consultation is an integral part of designing appropriate and acceptable fortification strategies, this study aimed to understand public awareness and perception of vitamin D fortified foods in the North East of England. A mixed methods approach was employed with two focus groups and a randomised survey of 109 participants from the Newcastle-upon-Tyne area. Thematic analysis was used to investigate focus group data, with factor and cluster analysis applied to establish differences in consumer perceptions and preferences.

Results indicate that participants had a good general awareness of vitamin D, with the majority (91\%) correctly identifying sunlight as the main source, although less were aware of the main dietary source (33\%), and fewer could state currently fortified products (51 $\%$ ). Interestingly, most participants did not perceive themselves as being at risk of vitamin D deficiency. Although attitudes towards fortification were favourable ( $63 \%$ agreed or strongly agreed that selected products should be fortified), nearly half (43\%) were unsure if more products should be made available, with a large majority $(73 \%)$ preferring to obtain vitamin D from natural sources. Factor analysis of 24 statements identified 7 factors, and subsequently 4 clusters each varying with knowledge of vitamin D and preferences for fortification practices. To have favourable purchase intentions towards fortified foods, participants needed to be aware of the health benefits associated with optimum vitamin D levels and the importance of, and ability to obtain vitamin D through dietary sources.

Results suggest that more natural products to complement existing market offerings would be most preferred. Overall the results indicate the importance of the level of awareness and knowledge of the health benefits associated with vitamin $\mathrm{D}$ to have favourable attitude towards fortified products, and potentially increase consumption. Nevertheless, in order to introduce successful fortification practices it is important to identify appropriate foods that reach the target population ${ }^{(3)}$.

1. Borrodale D \& Kimlin M (2009) Nutr Res Rev 22, 118-136.

2. O’Donnell S, Cranney A, Horsley T et al. (2008) Am J of Clin Nutr 88, 1528-1534.

3. Calvo MS, Whiting SJ \& Barton CN (2004) Am J of Clin Nutr 135, 310-316. 